\title{
Note on a Partition Function Which Assumes All Integral Values
}

\author{
Manvendra Tamba* \\ Department of Mathematics, Goa University, Taleigao Plateau, Goa, India \\ *Corresponding author: tamba@unigoa.ac.in \\ Received October 06, 2014; Revised November 17, 2014; Accepted November 27, 2014
}

\begin{abstract}
Let $G(n)$ denote the number of partitions of $n$ into distinct parts which are of the form $2 m, 3 m, 5 m, 6 m-3$, $8 m-3,9 m-3$ or $11 m-3$ with parts of the form $2 m, 3 m, 6 m-3$, or $11 m-3$ being even in number minus the number of them with parts of the form $2 m, 3 m, 6 m-3$, or $11 m-3$ being odd in number. In this paper, we prove that $G(n)$ assumes all integral values and does so infinitely often.
\end{abstract}

Keywords: partition functions, Gaussian integers, Jacobi's triple product identity

Cite This Article: Manvendra Tamba, "Note on a Partition Function Which Assumes All Integral Values." Turkish Journal of Analysis and Number Theory, vol. 2, no. 6 (2014): 220-222. doi: 10.12691/tjant-2-6-5.

\section{Introduction}

Let $S(n)$ denote the number of partitions of $n$ into distinct parts with even rank minus the number with odd rank (see [2]). Andrew, Dyson and Hickerson [3] used the arithmetic of $\mathrm{Q}(\sqrt{ } 6)$ to show that $\mathrm{S}(n)$ takes on every integral value infinitely often. This is the first time the interaction between the theory of partitions and algebraic number theory was exhibited. It was remarked in [3] that they know of no other partition function in the literature which assumes all integral values as $\mathrm{S}(\mathrm{n})$ does.

Let $\mathrm{H}(n)$ denote the number of partitions of $\mathrm{n}$ into parts which are repeated exactly $1,3,4,6,7,9$, or 10 times with the parts repeated exactly $1,4,6$, or 9 times even in number minus the number of them with parts repeated exactly 1,4 , 6 , or 9 times odd in number. In [5], using the arithmetic of Gaussian integers $\mathbb{Z}[\mathrm{i}]$, it was shown that $H(n)$ assumes all integral values and does so infinitely often.

Let $G(n)$ denote the number of partitions of $n$ into distinct parts which are of the form $2 \mathrm{~m}, 3 \mathrm{~m}, 5 \mathrm{~m}, 6 \mathrm{~m}-3$, $8 \mathrm{~m}-3,9 \mathrm{~m}-3$, or $11 \mathrm{~m}-3$ with parts of the form $2 \mathrm{~m}, 3 \mathrm{~m}, 6 \mathrm{~m}-$ 3 , or $11 \mathrm{~m}-3$ being even in number minus the number of them with parts of the form $2 \mathrm{~m}, 3 \mathrm{~m}, 6 \mathrm{~m}-3$, or $11 \mathrm{~m}-3$ being odd in number. For example, G(7) is zero because $(2(2))+(3(1)),(2(2))+(6(1)-3)$ have even number of parts of the form $2 \mathrm{~m}, 3 \mathrm{~m}, 6 \mathrm{~m}-3$, or $11 \mathrm{~m}-3$. while $(2(1))+(5(1))$ and $(2(1))+(8(1)-3)$ areodd number of parts of the form $2 \mathrm{~m}, 3 \mathrm{~m}, 6 \mathrm{~m}-3$, or $11 \mathrm{~m}-3$ (here $\mathrm{m}$-values are shown in bold). In this paper, we show that $G(n)$ assumes all integral values and does so infinitely often.

\section{Main Results}

A For (positive) integer $n$, consider the equation

$$
u^{2}+v^{2}=24 n+2
$$

We call a solution $(u, v)$ of (2.1) admissible if $u \equiv 1$ $(\bmod 6)$ and $v \equiv 1(\bmod 6)$. For a (positive) integer $n \equiv 2$ (mod 24), let $J(n)$ be the excess of the number of admissible solutions of $u^{2}+v^{2}=n$ with $v \equiv 1(\bmod 12)$ over the number of them with $v$ not congruent to 1 modulo 12.

In subsequent sections, we shall be proving the following:

Therorem 1. For $n \geq 0, G(n)=J(24 n+2)$.

Theorem 2. G(n) takes on every integer value infinitely often.

\section{Proof of Theorem 1}

First we note that the generating function of $G(n)$ is

$$
\begin{aligned}
& \sum_{n \geq 0} G(n) q^{n} \\
= & \prod_{n \geq 1}\left(\begin{array}{c}
1-q^{2 n}-q^{3 n}+q^{5 n}-q^{6 n-3} \\
+q^{8 n-3}+q^{9 n-3}-q^{11 n-3}
\end{array}\right)
\end{aligned}
$$

Lemma 1. For $|q|<1$,

$$
\begin{aligned}
& \prod_{n \geq 1}\left(\begin{array}{c}
1-q^{2 n}-q^{3 n}+q^{5 n}-q^{6 n-3} \\
+q^{8 n-3}+q^{9 n-3}-q^{11 n-3}
\end{array}\right) \\
& =\sum_{n, m \in \mathbb{Z}}(-1)^{m} q^{\frac{3}{2}\left(n^{2}+m^{2}\right)+\frac{1}{2}(n+m)}
\end{aligned}
$$

Proof. Using Jacobi's triple product identities (see [1], p. 21) we get 
$\sum(-1)^{m} q^{\frac{3}{2}}\left(n^{2}+m^{2}\right)+\frac{1}{2}(n+m)$

$n, m \in \mathbb{Z}$

$=\left(\sum_{n \in \mathbb{Z}} q^{\frac{3}{2} n^{2}+\frac{1}{2} n}\right)\left(\sum_{m \in \mathbb{Z}}(-1)^{m} q^{\frac{3}{2} m^{2}+\frac{1}{2} m}\right)$

$=\prod_{n \geq 1}\left(\left(1-q^{3 n}\right)\left(1+q^{3 n-1}\right)\left(1+q^{3 n-2}\right)\right)$

$\times \prod_{n \geq 1}\left(\left(1-q^{3 n}\right)\left(1-q^{3 n-1}\right)\left(1-q^{3 n-2}\right)\right)$

$=\prod_{n \geq 1}\left(\left(1-q^{3 n}\right)^{2}\left(1+q^{6 n-2}\right)\left(1+q^{6 n-4}\right)\right)$

$=\prod_{n \geq 1}\left(\left(1-q^{3 n}\right)\left(1-q^{2 n}\right)\left(1-q^{6 n-3}\right)\right)$

$=\prod_{n \geq 1}\left(\begin{array}{c}1-q^{2 n}-q^{3 n}+q^{5 n}-q^{6 n-3} \\ +q^{8 n-3}+q^{9 n-3}-q^{11 n-3}\end{array}\right)$

$=\sum_{n \geq 0} G(n) q^{n}$.

This proves the Lemma.

Using this lemma, it follows that:

$\sum_{n \geq 0} G(n) q^{24 n+2}$

$=q^{2} \prod_{n \geq 1}\left(\begin{array}{l}1-q^{48 n}-q^{72 n}+q^{120 n}-q^{144 n-72} \\ +q^{192 n-72}+q^{216 n-72}-q^{264 n-72}\end{array}\right)$

$=q^{2} \sum_{n, m \in \mathbb{Z}^{(-1)}} m^{36\left(n^{2}+m^{2}\right)+12(n+m)}$

$=\sum_{n, m \in \mathbb{Z}^{(-1)}}{ }^{m} q^{(6 n+1)^{2}+(6 m+1)^{2}}$

$=\sum_{n \geq 0} J(24 n+2) q^{24 n+2}$.

This proves Theorem 1 .

\section{Arithmetic of J(n)}

In this section we study $J(n)$ using Gaussian integers $\mathbb{Z}[i]$, where $i=\sqrt{-1}$. For $\alpha=u+i v \in \mathbb{Z}[i]$, let $N(u+i v)=u^{2}+v^{2}$. We define $c_{4}(\alpha)$ in terms of $u$ $(\bmod 4)$ and $v(\bmod 4)$ by

Table 1. Values of $c_{4}(\alpha)$

\begin{tabular}{|c|c|c|c|c|c|}
\hline \multirow{3}{*}{$u(\bmod 4)$} & \multicolumn{5}{|c|}{$v(\bmod 4)$} \\
\cline { 2 - 6 } & & 0 & 1 & 2 & 4 \\
\cline { 2 - 6 } & 1 & 1 & 0 & -1 & 0 \\
\cline { 2 - 6 } & 2 & 0 & $-i$ & 0 & $i$ \\
\cline { 2 - 6 } & 4 & -1 & 0 & 1 & 0 \\
\hline
\end{tabular}

Let $c_{\mathbf{3}}(\alpha)$ be defined in terms of $u(\bmod 3)$ and $v$ $(\bmod 3)$ by the following table, where $\omega=(1+i) / \sqrt{ } 2$ :
Table 2. Values of $c_{3}(\alpha)$

\begin{tabular}{|l|c|c|c|c|}
\hline \multirow{2}{*}{} & \multicolumn{5}{|c|}{$v(\bmod 3)$} \\
\hline \multirow{3}{*}{$u(\bmod 3)$} & & 0 & 1 & 2 \\
\cline { 2 - 5 } & 0 & 0 & $-i$ & $\mathrm{i}$ \\
\cline { 2 - 5 } & 1 & 1 & $\omega^{5}$ & $\omega^{7}$ \\
\cline { 2 - 5 } & 2 & -1 & $\omega^{3}$ & $\omega$ \\
\hline
\end{tabular}

Let $C(\alpha)=c_{3}(\alpha) c_{4}(\alpha)$ and let

$$
G(n)=\sum_{N(\alpha)=n} c(\alpha),
$$

where the sum is over a complex set non-associate Gaussian integers with norm $\mathrm{n}$.

Let $P=\{\alpha=u+i v \in \mathbb{Z}[i]: u \equiv 1(\bmod 6)$ and $v \equiv 1$ $(\bmod 6)\}$ and let $\mathrm{Q}=\{\beta=r+i s \in \mathbb{Z}[i]:(1+i) \beta P$ or $(1-i) \beta \in P\}$. Then, for $n \equiv 1(\bmod 12)$,

$$
J(2 n)=\sum_{N(\alpha)=2 n}^{\alpha \in P}(-1)^{(\nu-1) / 2},
$$

(where $v$ is the imaginary part of $\alpha$ )

$$
=\sum_{N(\beta)=n}^{\beta \in Q}(c(\beta)+c(\bar{\beta})),
$$

(where $\bar{\beta}$ is the conjugate of $\beta$ )

$=C(n)$.

Note that this together with Theorem 1 proves the assertion made in the Remark 2 of [5].

Thus we have shown that:

Lemma 2. For $n \equiv 1(\bmod 12), J(2 n)=C(n)$.

Next we recall the properties of $\mathrm{C}(\mathrm{n})$ from [5].

Lemma 3. (a) The function $\mathrm{C}(\mathrm{n})$ is multiplicative.

(b) $C(n)=0$ unless $n \equiv 1$ or $5(\bmod 12)$.

Lemma 4. Let $p$ be a prime $\equiv 1(\bmod 12)$ and $n \geq 1$. Then:

a. $\mathrm{C}(\mathrm{n})$ is either 0,2 or -2 .

b. If $C(p)=0$, then

$$
C\left(p^{n}\right)=\left\{\begin{array}{cl}
(-1)^{n / 2} & \text { if niseven } \\
0 & \text { other wise }
\end{array}\right.
$$

c. If $C(p)= \pm 2$, then

$$
\left(p^{n}\right)=\left\{\begin{array}{cc}
(n+1) & \text { if } C(p)=2 \\
(-1)^{n}(n+1) & \text { if } C(p)=-2
\end{array}\right.
$$

Lemma 5. Let $p$ be a prime $\equiv 5(\bmod 12)$ and $n \geq 1$ be even. Then:

$$
C\left(p^{n}\right)=\left\{\begin{array}{cc}
(-1)^{n / 4} & \text { if } n \equiv 0(\bmod 4) \\
(-1)^{(n+2) / 4} & \text { if } n \equiv 2(\bmod 4) .
\end{array}\right.
$$

\section{Proof of Theorem 2}

As in [5], 
$G\left(\frac{61^{k-1} 13^{2 m}-1}{12}\right)=C\left(61^{k-1} \cdot 13^{2 m}\right)$

(by Theorem 1and Lemma 2)

$=C\left(61^{k-1}\right) C\left(13^{2 m}\right) \quad($ by Lemma $3(\mathrm{a}))$

$=k(-1)^{m}$

(by Lemma 4 (b) and (c)

and [[5], Table 1])

$$
=\left\{\begin{array}{cl}
k & \text { if } m \text { is even } \\
-k & \text { if } m \text { is odd } .
\end{array}\right.
$$

This proves Theorem 2 .

\section{Conclusion}

An arithmetical function $f(n)$ is called lacunary if it is almost always 0 (see[4]). In [3] it is shown that $S(n)$ is lacunary. In [5] it is shown that $H(n)$ is lacunary. So is is natural to ask whether $G(n)$ is so. We make the following conjecture:

Conjecture. $G(n)$ is lacunary.

\section{References}

[1] G.E. Andrews, The Theory of Partitions, (G.-C. Rota, Ed.), Encyclopedia of Math. And its Applications, Vol. 2, AddisonWesley, Reading, MA, 1976.

[2] G.E. Andrews, Questions and conjectures in partition theory, Amer. Math. Monthly, 93 (1986) 708-711.

[3] G.E. Andrews, F.J. Dyson and D. Hickerson, Partitions and indefinitequadratic forms, Invent. math., 91 (1988) 391-407.

[4] J. Lovejoy, Lacunary Partition Functions, Math. Research Letters, 9 (2002) 191-198.

[5] M.Tamba, On a partition function which assumes all integral values, J.Number Theory 41 (1992) 77-86. 